\title{
Brain-Derived Neurotrophic Factor Mediates Activity- Dependent Dendritic Growth in Nonpyramidal Neocortical Interneurons in Developing Organotypic Cultures
}

\author{
Xiaoming Jin, ${ }^{1}$ Hang Hu, ${ }^{1}$ Peter H. Mathers, ${ }^{2,3,4,5}$ and Ariel Agmon ${ }^{1,5}$ \\ Departments of ${ }^{1}$ Neurobiology and Anatomy, ${ }^{2}$ Otolaryngology, ${ }^{3}$ Biochemistry and Molecular Pharmacology, and ${ }^{4}$ Ophthalmology, and ${ }^{5}$ The Sensory \\ Neuroscience Research Center, West Virginia University, Morgantown, West Virginia 26506-9128
}

\begin{abstract}
Brain-derived neurotrophic factor (BDNF) promotes postnatal maturation of GABAergic inhibition in the cerebral and cerebellar cortices, and its expression and release are enhanced by neuronal activity, suggesting that it acts in a feedback manner to maintain a balance between excitation and inhibition during development. BDNF promotes differentiation of cerebellar, hippocampal, and neostriatal inhibitory neurons, but its effects on the dendritic development of neocortical inhibitory interneurons remain unknown. Here, we show that BDNF mediates depolarization-induced dendritic growth and branching in neocortical interneurons. To visualize inhibitory interneurons, we biolistically transfected organotypic cortical slice cultures from neonatal mice with green fluorescent protein (GFP) driven by the glutamic acid decarboxylase (GAD)67 promoter. Nearly all GAD67-GFP-expressing neurons were nonpyramidal, many contained GABA, and some expressed markers of neurochemically defined GABAergic subtypes, indicating that GAD67-GFP-expressing neurons were GABAergic. We traced dendritic trees from confocal images of the same GAD67-GFP-expressing neurons before and after a $5 \mathrm{~d}$ growth period, and quantified the change in total dendritic length (TDL) and total dendritic branch points (TDBPs) for each neuron. GAD67-GFP-expressing neurons growing in control medium exhibited a $20 \%$ increase in TDL, but in $200 \mathrm{ng} / \mathrm{ml} \mathrm{BDNF}$ or $10 \mathrm{~mm} \mathrm{KCl}$, this increase nearly doubled and was accompanied by a significant increase in TDBPs. Blocking action potentials with TTX did not prevent the BDNF-induced growth, but antibodies against BDNF blocked the growth-promoting effect of KCl. We conclude that BDNF, released by neocortical pyramidal neurons in response to depolarization, enhances dendritic growth and branching in nearby inhibitory interneurons.
\end{abstract}

Key words: biolistic; BDNF; GABA; GAD67; gene gun; inhibitory interneuron; neocortical development; neurotrophins; organotypic slice

\section{Introduction}

GABAergic inhibition in the rodent cerebral cortex, although already functional at birth (Agmon et al., 1996; Wells et al., 2000), exhibits a protracted period of postnatal maturation lasting for several weeks (Komatsu and Iwakiri, 1991; Agmon and O'Dowd, 1992; Sutor and Luhmann, 1995; Cohen et al., 2000). Some of the developmental changes in inhibition occur postsynaptically, but others are presynaptic and include biochemical, morphological, and electrophysiological differentiation of GABAergic interneurons (Miller, 1986; Balcar et al., 1992; Micheva and Beaulieu, 1995; Guo et al., 1997; Massengill et al., 1997) and formation of GABAergic synapses (Micheva and Beaulieu, 1996; De Felipe et al., 1997; Marty et al., 2002). The delayed maturation of inhibi-

\footnotetext{
Received Oct. 1, 2002; revised May 5, 2003; accepted May 5, 2003.

This work was supported by National Institutes of Health Grants HD33463 (A.A.) and EY12152 (P.H.M.). We thank Albert Berrebi for helpful advice on immunocytochemistry and comments on this manuscript. We also thank Janet Cyr and Jason Wells for helpful comments on this manuscript; Cary Johnson, Jeff Altemus, Colette Ramsburg, and Eric Christenson for excellent technical support; and Dr. Gabor Szabo for providing the GAD67 promoter.

Correspondence should be addressed to Dr. Aric Agmon, Department of Neurobiology and Anatomy, West Virginia University, Health Science Center Drive, Morgantown, WV 26506-9128. E-mail: aagmon@wvu.edu.

$X$. Jin's present address: Department of Neurology and Neurological Sciences, Stanford University School of Medicine, Stanford, CA 94305

Copyright $\odot 2003$ Society for Neuroscience $\quad$ 0270-6474/03/235662-12\$15.00/0
}

tion may allow increased NMDA receptor activation in the neonatal cortex, thereby opening a developmental window of enhanced plasticity during an early postnatal critical period (Agmon and O’Dowd, 1992; Kirkwood and Bear, 1994; Ramoa and McCormick, 1994; Rozas et al., 2001).

An attractive hypothesis that accounts for the delayed maturation of GABAergic inhibition suggests that inhibition is upregulated by neuronal excitation, thus establishing a negative feedback loop that counteracts the developmental increase in glutamatergic excitation, albeit with a lag (Marty et al., 1997; Bolton et al., 2000; Turrigiano and Nelson, 2000). A putative mediator of various effects of electrical activity is brain-derived neurotrophic factor (BDNF), a neurotrophin acting on tyrosine kinase (Trk)B receptors and implicated in neuronal development and synaptic plasticity (Thoenen, 1995; Bonhoeffer, 1996; McAllister et al., 1999; Lu, 2003). In the cerebellum and hippocampus, activity-induced enhancement of GABAergic mechanisms can be mimicked by exogenous BDNF and/or prevented by BDNF inhibitors (Marty et al., 1996b, 2000; Rutherford et al., 1997; Seil and Drake-Baumann, 2000), suggesting that BDNF may also mediate activity-dependent maturation of inhibition. Consistent with this hypothesis, GABAergic maturation is accelerated in the cerebellum and cerebral cortex of BDNF-overexpressing mice 
(Bao et al., 1999; Huang et al., 1999; Aguado et al., 2003). Conversely, GABAergic maturation is retarded in the cerebellum and cerebral cortex of TrkB knock-out mice (Rico et al., 2002; Carmona et al., 2003) and in the cerebellum of the stargazer mouse, a mutant that is deficient in BDNF (Richardson and Leitch, 2002) [but see Olofsdotter et al. (2000) and Henneberger et al. (2002) for the apparently opposite result of enhanced inhibition in the dentate gyrus and superior colliculus of BDNF knock-out mice].

BDNF promotes neurochemical and dendritic differentiation of inhibitory neurons of the cerebellum, neostriatum, and hippocampus (Nawa et al., 1994; Ventimiglia et al., 1995; Marty et al., 1996a; Vicario-Abejon et al., 1998; Mertz et al., 2000), but its effects on dendritic growth of GABAergic interneurons in the neocortex remain unexplored. Here, we used a novel preparation (Jin et al., 2001) to follow the long-term dendritic development of individual GFP-expressing neocortical interneurons in organotypic cultures. We found that both BDNF and depolarization enhanced dendritic growth in nonpyramidal neocortical interneurons, and that the effect of depolarization was dependent on the release of endogenous BDNF.

\section{Materials and Methods}

Organotypic culture preparation. Organotypic cortical slice cultures were prepared as described previously (Stoppini et al., 1991; Jin et al., 2001). Postnatal day 1-3 mouse pups were anesthetized by cooling and decapitated, and their brains were rapidly removed and immersed in ice-cold artificial CSF (ACSF) (containing in mM: $126 \mathrm{NaCl}, 3.0 \mathrm{KCl}, 1.3 \mathrm{MgSO}_{4}$, $2.5 \mathrm{CaCl}_{2}, 1.2 \mathrm{NaH}_{2} \mathrm{PO}_{4}, 26 \mathrm{NaHCO}_{3}$, and 20 dextrose, saturated with $95 \% \mathrm{O}_{2}-5 \% \mathrm{CO}_{2}$ ). Under sterile conditions in a laminar flow hood, 350 $\mu \mathrm{m}$-thick coronal brain slices of parietal cortex were cut with a Vibroslice (World Precision Instruments, Sarasota, FL). Slices were then placed on $30 \mathrm{~mm}$ Millicell membrane inserts (Millipore, Bedford, MA), usually two slices per insert, and the inserts were placed in individual wells of six-well plates containing $1 \mathrm{ml}$ of culture medium. The culture medium was composed of the following (in $\mathrm{ml}$ per $100 \mathrm{ml}$ ): 46 Eagle's basal medium, 25 Earle's balanced salt solution, 25 horse serum, 1 mixture of penicillin $(10,000 \mathrm{U} / \mathrm{ml})$-streptomycin $(10 \mathrm{mg} / \mathrm{ml})$-glutamine $(29.2 \mathrm{mg} / \mathrm{ml})$ (all from Invitrogen, Carlsbad, CA), and $320 \%$ glucose solution. The slices were kept in a humidified incubator at $35^{\circ} \mathrm{C}$ with a $5 \% \mathrm{CO}_{2}$-enhanced atmosphere, and the medium was changed twice per week. After $2-3 \mathrm{~d}$ in culture, slices were transfected using a Helios gene gun (Bio-Rad, Hercules, CA).

Gene gun-mediated transfection. GAD67-GFP plasmid DNA was cloned from a $10.3 \mathrm{~kb}$ segment of the mouse GAD67 promoter region (Szabo et al., 1996; Katarova et al., 1998) fused in-frame to enhanced GFP (Clontech, Palo Alto, CA), as described previously (Jin et al., 2001). Cytomegalovirus (CMV)-GFP plasmid DNA (pGreen Lantern) was purchased from Invitrogen. Gene gun cartridges were prepared according to the manufacturer's protocol with slight modifications: for each 25 inch plastic tube, $12 \mathrm{mg}$ of gold particles (1.0 or $1.6 \mu \mathrm{m}$ diameter) and $25 \mu \mathrm{g}$ of plasmid DNA $(1 \mu \mathrm{g} / \mu \mathrm{l}$ in distilled water) were mixed in $100 \mu \mathrm{l}$ of 50 mM spermidine, precipitated with $100 \mu \mathrm{l}$ of $1 \mathrm{M} \mathrm{CaCl}_{2}$, washed three times in $100 \%$ ethanol, and then resuspended in $3 \mathrm{ml}$ of $0.02 \mathrm{mg} / \mathrm{ml}$ polyvinylpyrrolidone in ethanol and precipitated onto the internal wall of the plastic tube. Slices were bombarded with one cartridge per insert under 120 psi helium pressure through a nylon mesh that served to reduce the mechanical effects of the gas blast.

Confocal microscopy. Confocal images were first acquired at least $2 \mathrm{~d}$ after gene gun transfection to allow complete GFP filling of distal dendrites. Cultured brain slices were removed by cutting out the underlying Millicell membrane (leaving it adherent to the culture) and placed under sterile conditions in an imaging chamber filled with freshly oxygenated ACSF at room temperature. The imaging chamber was made of two round glass coverslips separated by $4 \mathrm{~mm}$ and held by a stainless-steel frame with a $17 \mathrm{~mm}$-wide circular opening to allow imaging. Well separated neurons with bright GFP expression were selected for imaging. Neurons were imaged with a $20 \times, 0.7$ numerical aperture (NA) dry objective on an inverted Zeiss (Thornwood, NY) LSM 510 laser-scanning confocal microscope, using the $488 \mathrm{~nm}$ argon laser line and a 505-550 $\mathrm{nm}$ bandpass emission filter. Image stacks (typically $10-20$ optical sections per stack) were collected at $1.5-2.5 \mu \mathrm{m} z$-axis steps through the full extent of the dendritic tree and saved for off-line tracing and analysis. Images were taken with the lowest practical laser intensity and the shortest practical illumination time to limit photodynamic damage, and slices were kept outside the incubator for $<1 \mathrm{hr}$ per session. This imaging protocol was found not to cause any apparent ill effects to the imaged neurons or the slice culture, because most neurons remained viable and exhibited the same general dendritic morphology when imaged again 5 or $10 \mathrm{~d}$ later (see Fig. 3), and some remained viable even after repeated imaging over several weeks (data not shown). At the end of each imaging session, low-power $(10 \times)$ images were taken in fluorescence and transmission modes to record the locations of the GFP-expressing neurons in the cortex relative to the pia and white matter. The slices with their adherent membranes were then placed back into multiwell plates under sterile conditions and returned to the incubator. The same neurons were imaged again $5 \mathrm{~d}$ later.

Pharmacological treatments. In some slices, one or more of the following were added to the culture medium: $200 \mathrm{ng} / \mathrm{ml}$ recombinant human BDNF (Alomone Labs, Jerusalem, Israel), $250 \mathrm{ng} / \mathrm{ml}$ recombinant human neurotrophin (NT)4/5 (courtesy of Genentech, South San Francisco, CA), $50 \mu \mathrm{g} / \mathrm{ml}$ rabbit anti-BDNF polyclonal antibody (Chemicon, Temecula, CA), $200 \mathrm{~nm} \mathrm{K252a}$, and $1 \mu \mathrm{m}$ tetrodotoxin (TTX) (both from Alomone Labs). Treatments were refreshed on medium change. Pharmacological treatments started the day after the first imaging session; therefore, treatments lasted 4 of the $5 \mathrm{~d}$ between imaging sessions. Control experiments (slices cultured in normal medium) were interspersed throughout the treatment experiments.

Biocytin filling and histochemistry. To verify that GFP fluorescence revealed the full dendritic morphology, a subset of GFP-expressing neurons were filled with biocytin after confocal imaging. For biocytin filling, slices with the underlying Millicell membranes were transferred to a submersion recording chamber and continuously superfused with ACSF saturated with $95 \% \mathrm{O}_{2}-5 \% \mathrm{CO}_{2}$ at room temperature. GFP-expressing neurons were visualized with a $40 \times, 0.8 \mathrm{NA}$ water immersion objective under an Olympus Optical (Melville, NY) BX50 upright microscope equipped with fluorescence and differential interference contrast optics and a Hamamatsu (Bridgewater, NJ) CCD camera. Patch pipettes were pulled from $1.5 \mathrm{~mm}$ outer diameter glass capillary tubes (World Precision Instruments) and filled with a solution of $136 \mathrm{~mm}$ potassium gluconate, $2 \mathrm{~mm} \mathrm{MgCl}_{2}, 0.6 \mathrm{~mm}$ EGTA, $10 \mathrm{~mm}$ HEPES, $2 \mathrm{mg} / \mathrm{ml}$ biocytin, with $\mathrm{pH}$ adjusted to 7.3 and osmolarity to $275-285 \mathrm{mOsm}$. A whole-cell clamp configuration was achieved using the Axopatch $200 \mathrm{~A}$ patch-clamp amplifier (Axon Instruments, Foster City, CA) and maintained for $\sim 1 \mathrm{hr}$ to allow for complete diffusion of biocytin. Slices were then fixed overnight in $0.1 \mathrm{M}$ PBS with $4 \%$ paraformaldehyde at $4^{\circ} \mathrm{C}$. After three rinses in PBS, fixed slices were incubated for $2 \mathrm{hr}$ with $\mathrm{ABC}$ solution (Vector Laboratories, Burlingame, $\mathrm{CA}$ ), followed by additional rinses in $\mathrm{PBS}$ and staining with diaminobenzidine $(\mathrm{DAB})(0.7 \mathrm{mg} / \mathrm{ml})$ and $\mathrm{H}_{2} \mathrm{O}_{2}(0.3 \%)$ in PBS. The reaction was stopped by transferring the slices to cold PBS.

Immunocytochemistry. For immunocytochemistry, GAD67-GFPtransfected slices were fixed for $\sim 1 \mathrm{hr}$ at room temperature and then overnight at $4^{\circ} \mathrm{C}$ in $4 \%$ paraformaldehyde in PBS, rinsed three times in PBS, and blocked for 1-3 hr in PBS containing 5-10\% normal horse serum and $0.5-1 \%$ Triton X-100. Slices were then incubated for $2-3 \mathrm{~d}$ at $4{ }^{\circ} \mathrm{C}$ with one or two of the following antibodies (at 1:1000 in PBS containing $1-5 \%$ normal horse serum and $0.1-0.5 \%$ Triton X-100): rabbit anti-GAD (AB108; Chemicon), mouse monoclonal anti-parvalbumin (P3088; Sigma, St. Louis, MO), mouse monoclonal anti-calbindin D28k (AB1778; Chemicon), rabbit polyclonal anti-calretinin (AB149; Chemicon), rabbit polyclonal anti-somatostatin (T-4103; Peninsula Laboratories, San Carlos, CA), rabbit polyclonal anti-neuropeptide Y (T-4070; Peninsula Laboratories), and rabbit polyclonal anti-GABA [gift of David Pow (University of Queensland, St. Lucia, Australia)] [this antibody was designed for paraformaldehyde-only fixation, and its specificity was established previously (Pow et al., 1995; Spirou and Berrebi, 1997)]. After incubation with antibody, slices were washed three times in PBS, fol- 


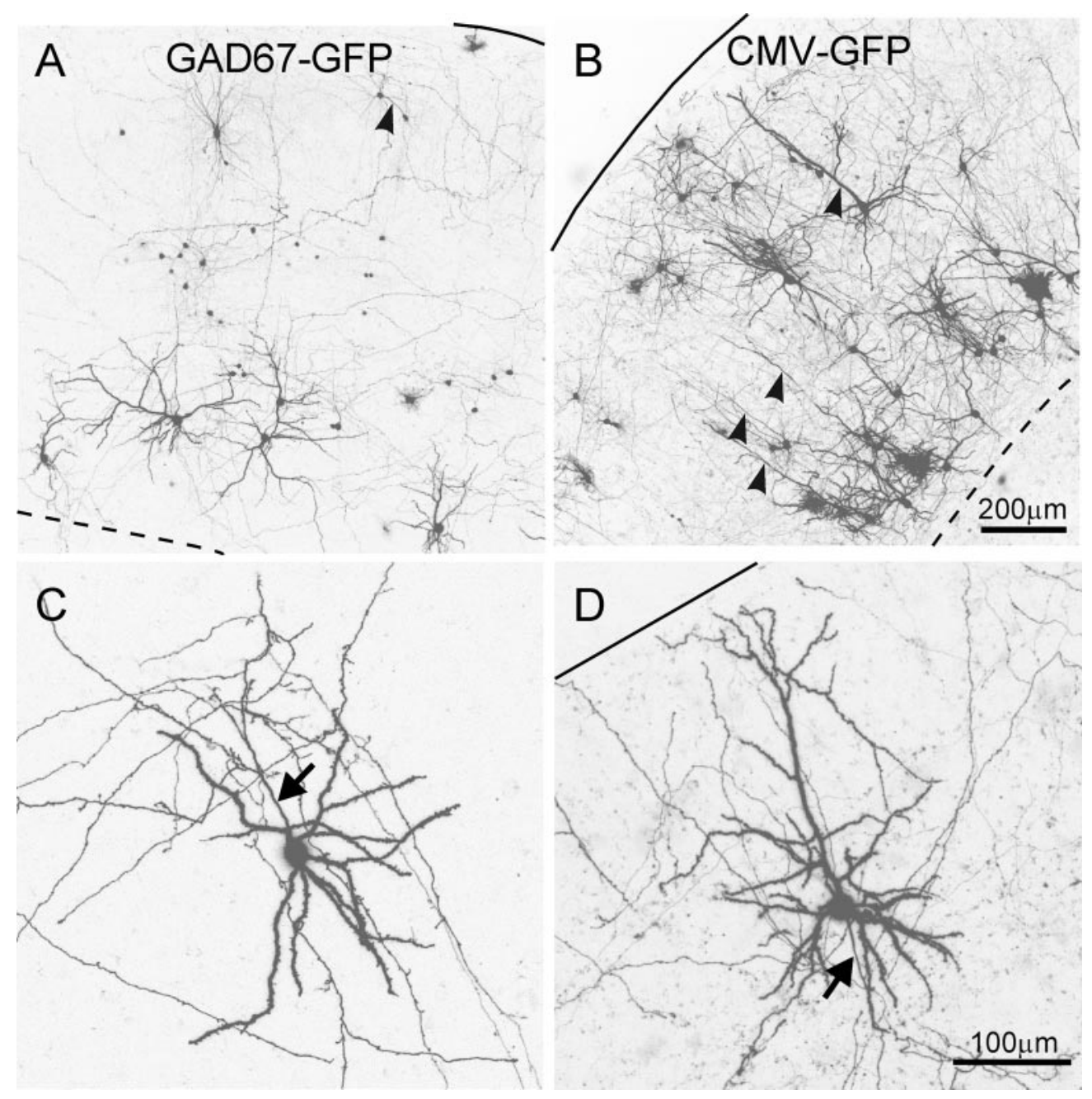

Figure 1. Neurons expressing GAD67-GFP were nonpyramidal, but neurons expressing CMV-GFP had mixed morphologies. A, C, Neurons labeled by transfection with GAD67-GFP, imaged at low $(10 \times)$ and high $(20 \times)$ power, respectively. $B, D$, Neurons labeled by transfection with CMV-GFP, imaged at low and high power, respectively. The images in $A-D$ are from four different slices. Arrowhead in $A$ points to a pair of apparently fused neurons; arrowheads in $B$ indicate apical dendrites of pyramidal neurons. Arrows in $C$ and $D$ indicate the initial segment of the axon, which exits toward the pial surface in the interneuron $(C$ and toward the white matter in the pyramidal neuron $(D)$. Solid lines in this and subsequent figures indicate the pial surface, and dashed lines indicate the border between layer 6 and the white matter. Scale bars: (in $B) A, B, 200 \mu \mathrm{m}$; (in D) C, D, $100 \mu \mathrm{m}$.

lowed by one or both of the following secondary antibodies (at 1:1000 in PBS with $1 \%$ normal horse serum): Alexa 546 goat anti-rabbit $\operatorname{IgG}\left(\mathrm{ab}^{\prime}\right)_{2}$ fragments or Alexa 633 goat anti-mouse $\operatorname{IgG}\left(\mathrm{ab}^{\prime}\right)_{2}$ fragments (both from Molecular Probes, Eugene, OR), for $2 \mathrm{hr}$ at room temperature. The slices were then washed three times in PBS, mounted in Vectashield (Vector Laboratories), and coverslipped.

Neuronal tracing. Neurons with strong GFP fluorescence throughout their dendritic tree were reconstructed digitally from the confocal image stacks using Neurolucida software (MicroBrightField, Colchester, VT). GFP-expressing neurons that were filled with biocytin were traced a second time with Neurolucida after processing the DAB reaction, using a $20 \times, 0.7$ NA objective and a CCD video camera attached to an Olympus Optical AX70 microscope.

Data analysis. Quantitative analysis on the traced data were done using Neuroexplorer software (MicroBrightField). Numerical data (total dendritic length and branch points) generated from the second imaging session (day 5 ) were divided by the values derived from the first imaging session (day 0) to yield a ratio; geometrical means and SEs of the ratios were then calculated for each treatment group and for the control group.

Statistics. Exact, distribution-independent permutation tests were used to compare experimental treatments with control (Manly, 1997; Good, 1999). Computations were done using MathCad (MathSoft, Cambridge, MA). When a group mean was compared with an expected value, the binomial sign test (Fisher's one-sample randomization test) was used: each data point was assigned a positive or a negative sign on the basis of its value relative to the expected mean, and the probability that an equal or more extreme distribution of signs would occur at random was calculated from the binomial probability function. When the means of two groups were compared, the difference between the means was calculated for 10,000 random permutations of the data, and the fraction of values equal to or more extreme than the experimental observation was doubled to yield a two-tailed $p$ value. All of the comparisons were made pairwise, in general between each experimental group and the control group, but in some cases (indicated) between two experimental groups, with no adjustments for multiple comparisons (Rothman, 1990; Savitz and Olshan, 1998).

\section{Results}

\section{Transfecting GABAergic interneurons with GAD67-GFP}

To visualize nonpyramidal neocortical interneurons, we transfected cortical organotypic slices with a DNA construct in which the GAD67 promoter drives the expression of GFP (Jin et al., 2001), because GAD is a high-fidelity marker for GABAergic neurons (Mugnaini and Oertel, 1985). Two days after transfection, up to a few dozen neurons per slice expressed GFP fluorescence at varying levels of intensity (Fig. $1 A$ ). In strongly fluorescent neurons, minute morphological details were discernible, including 
dendritic filopodia and spines and fine axonal arborizations (Fig. $1 C)$. GABAergic neurons in the cerebral cortex exhibit a nonpyramidal morphology (Houser et al., 1983; Meinecke and Peters, 1987; Prieto et al., 1994); therefore, to confirm that GAD67-GFP expression is restricted to GABAergic interneurons, we compared the morphology of GAD67-GFP-expressing neurons with that of neurons transfected with a GFP construct driven by the nonspecific CMV promoter (CMV-GFP) (Fig. $1 B$ ). Neocortical neurons with a long vertical dendrite extending toward the pia and several additional primary dendrites growing in all of the other directions were defined as pyramidal-like (Fig. $1 D$ ), and all of the other neurons were defined as nonpyramidal. In GAD67GFP-transfected slices, 92\% of GFP-expressing neurons were nonpyramidal ( $n=212$ neurons) (Fig. $1 A, C$ ); in contrast, CMV-GFP-expressing neurons were divided about evenly between pyramidal (45\%) and nonpyramidal (55\%) morphologies $(n=71)$ (Fig. $1 B, D)$. The difference between slices transfected with GAD67-GFP and CMV-GFP was highly significant $(p<$ $10^{-10}$; Fisher's exact test). The higher percentage of nonpyramidal neurons in CMV-GFP-transfected slices compared with the known incidence of GABAergic neurons in the rodent neocortex (15-25\%) (Ren et al., 1992; Micheva and Beaulieu, 1995) could have reflected a higher efficacy of the CMV promoter in GABAergic neurons, although misclassification of some pyramidal neurons as nonpyramidal because of truncation of their apical dendrites during the slicing procedure, or CMV-GFP expression by spiny stellate cells (layer 4 neurons that are glutamatergic but nonpyramidal), cannot be ruled out. Nevertheless, the small fraction $(8 \%)$ of neurons with pyramidal-like morphology among hundreds of GAD67-GFP-expressing cells strongly suggests that GAD67-GFP expression was primarily restricted to GABAergic interneurons. GAD67-GFP-expressing cells with pyramidal-like morphology were excluded from additional study.

In many slices, a small number of GFP-expressing neurons were observed to form clusters of two to three closely situated cells that appeared to be fused together at a single contact point on their somata or their proximal dendrites (Fig. $1 A$, arrowhead). Because such fused neurons were found in both GAD67-GFPand CMV-GFP-transfected slices, we assume that the fusion was induced by the mechanical effect of the biolistic transfection; however, the precise mechanism remains to be determined. Fused neurons were excluded from additional study.

As an additional test of the neurotransmitter content of GAD67-GFP-expressing neurons, we stained them immunocytochemically with antibodies to GABA (Fig. $2 A, B$ ) and to GAD67 (Fig. 2C,D). Forty-two percent of GAD67-GFP-expressing neurons examined were immunopositive for GABA $(n=24)$, but only $26 \%$ were immunopositive for GAD67 $(n=34)$. Moreover, GAD67-GFP-expressing neurons staining immunopositive for either GABA or GAD67 were among the least intensely stained neurons in their microscopic field (Fig. $2 B, D$ ). This could reflect a low level of GAD67 (and therefore GABA) expression in GFPexpressing neurons, possibly because of competition for limited transcription factors between the endogenous and exogenous GAD67 promoters.

Cortical GABAergic interneurons can be classified into several different subtypes with distinct neurochemical identities (Kubota et al., 1994; Kawaguchi and Kubota, 1997). To test whether biolistic transfection with GAD67-GFP preferentially labeled GABAergic interneurons from one or more of these classes, we immunostained GAD67-GFP-transfected slices for the calciumbinding proteins parvalbumin (PV), calbindin (CB), and calretinin (CR) and for the neuropeptides somatostatin (SOM) and neuropeptide Y (NPY). In this analysis, we also included GAD67-GFP-expressing neurons in the hippocampus, because the neurochemical composition of the hippocampus is virtually identical to that of the neocortex, and because many of the GABAergic interneuron classes in the hippocampus can be readily recognized on the basis of their laminar position and morphological features (Freund and Buzsaki, 1996). Between 4 and $12 \%$ of GAD67-GFP-expressing neurons coexpressed any one of these markers (PV, 7.4\% of 189 cells; CB, $6.7 \%$ of 74 cells; CR, $4 \%$ of 51 cells; SOM, $12.1 \%$ of 157 cells; NPY, $4 \%$ of 25 cells).

The incidence of GABAergic subtype markers in GAD67GFP-expressing neurons was well below the incidence of the same markers in the GABAergic population of the mature cortex in vivo; for example, approximately one-half of all of the GABAergic neurons are estimated to express PV in the adult rat neocortex (Ren et al., 1992). Our immunostaining experiments, however, were done on slices younger than 2 weeks of equivalent age (we define equivalent age as postnatal day at culturing plus days in vitro), when PV mRNA and protein are just becoming detectable in vivo (Alcantara et al., 1993; del Rio et al., 1994; de Lecea et al., 1995). Moreover, PV expression is considerably reduced in neocortical slice cultures prepared from neonatal animals, even after several weeks in culture (Vogt Weisenhorn et al., 1998).

Unlike parvalbumin, globally reduced expression could not underlie the low incidence of somatostatin in GAD67-GFPexpressing cells, as demonstrated by two contrasting examples in Figure $2 E-H$. The large GFP-expressing neuron in Figure $2 E$, with a cell body at the oriens-alveus border of CA1, was by its laminar location and dendritic morphology a typical oriens-lacunosum moleculare interneuron, a class of hippocampal interneurons known to express SOM (Freund and Buzsaki, 1996), and was indeed SOM immunopositive (Fig. $2 F$ ). In contrast, the GFP-expressing neuron in Figure $2 G$, with its cell body in cortical layer 6, was by its ascending axons (inset) an unmistakable Martinotti cell, a class of cortical interneurons also known to contain SOM (Wahle, 1993; Kawaguchi and Kubota, 1996); however, this cell was SOM immunonegative (Fig. $2 H$ ), although several nonGFP-expressing neurons in the same microscopic field were clearly immunopositive. These examples show that the low incidence of somatostatin in GAD67-GFP-expressing neurons did not result from a general low level of immunoreactivity to this peptide, nor could it be attributable to an inadvertent inclusion in our sample of glutamatergic neurons. Most likely, it reflected lower levels of SOM in GAD67-GFP-expressing neurons belonging to this GABAergic subtype, possibly because of diversion of their biochemical resources to the production of large amounts of an exogenous protein (GFP). It should be noted that, in mice carrying a GAD67-GFP transgene, neurons coexpressing GFP and SOM are often less intensely stained (for SOM) than nonGFP-expressing, SOM-immunopositive neurons nearby (our unpublished observations), indicating similar competitive pressures in transgenic animals, although the transgene is presumably present in the genome of transgenic mice in far fewer copies than those of the GAD67-GFP construct in biolistically transfected neurons.

\section{Dendritic development of nonpyramidal cortical interneurons in organotypic slice cultures}

Because GFP expression in our cultures revealed the detailed morphology of living neurons, we were able to follow the morphological development of individual neurons over time by imaging the same neuron two or more times at $5 \mathrm{~d}$ intervals, digitally 
reconstructing the three-dimensional (3-D) dendritic morphology of the neuron from stacks of confocal images, and quantifying the TDL and TDBPs at each imaging time point.

We first followed the growth of GFPexpressing neurons in normal medium (Fig. 3). When first imaged, the age of our cultures varied between equivalent postnatal day 7 (EP7) (equivalent postnatal day $=$ postnatal day at culturing + days in vitro) and EP24. We quantified dendritic growth over the next $5 \mathrm{~d}$ by calculating the ratio between TDL at second imaging (day 5 ) and TDL at first imaging (day 0). This ratio is plotted in Figure $3 C$ against equivalent age at first imaging. The mean TDL ratio was $1.22 \pm 0.07$ ( $n=19$ neurons $)$ for neurons first imaged at EP10 or earlier (left of vertical dotted line), but was $1.01 \pm$ $0.03(n=26)$ for neurons first imaged at older ages (right of dotted line). The difference between the means of the two groups was highly significant $(p=0.0008)$. Notably, the SD of the TDL ratios in the younger group was twice as large as in the older group, indicating a higher variability in growth rate in younger slices. As an additional measure of growth, we also compared the TDBP ratio of the second to the first imaging. The mean TDBP ratio $(0.93 \pm 0.09$ and $1.03 \pm 0.06$, respectively, for the younger and older groups) was not significantly different from 1 for either group ( $p=0.5$ and 0.18 , respectively), indicating that, in our control conditions, there was a balance between the addition and elimination of new branches, even when (as in the younger age group) there was a net elongation of dendrites. These data suggest that dendritic growth in our nonpyramidal cortical interneurons occurred mostly during the first 2 weeks in organotypic culture, and that dendritic branching patterns may have been already fully established by the age of our earliest imaging (EP7). Because our goal was to study the role of BDNF during normal development, all of the subsequent experiments were done on the younger age group (EP7-EP10 at first imaging).

Because organotypic slices grow thinner and more translucent with time in culture, and because the level of GFP expression often appeared to increase with time in culture, we were concerned that any apparent increase in dendritic length between the two imaging sessions could be the result of incomplete visualization or incomplete GFP filling of dendrites at the earliest imaging time point. To test this possibility, we compared dendritic filling and visualization between GFP and biocy-

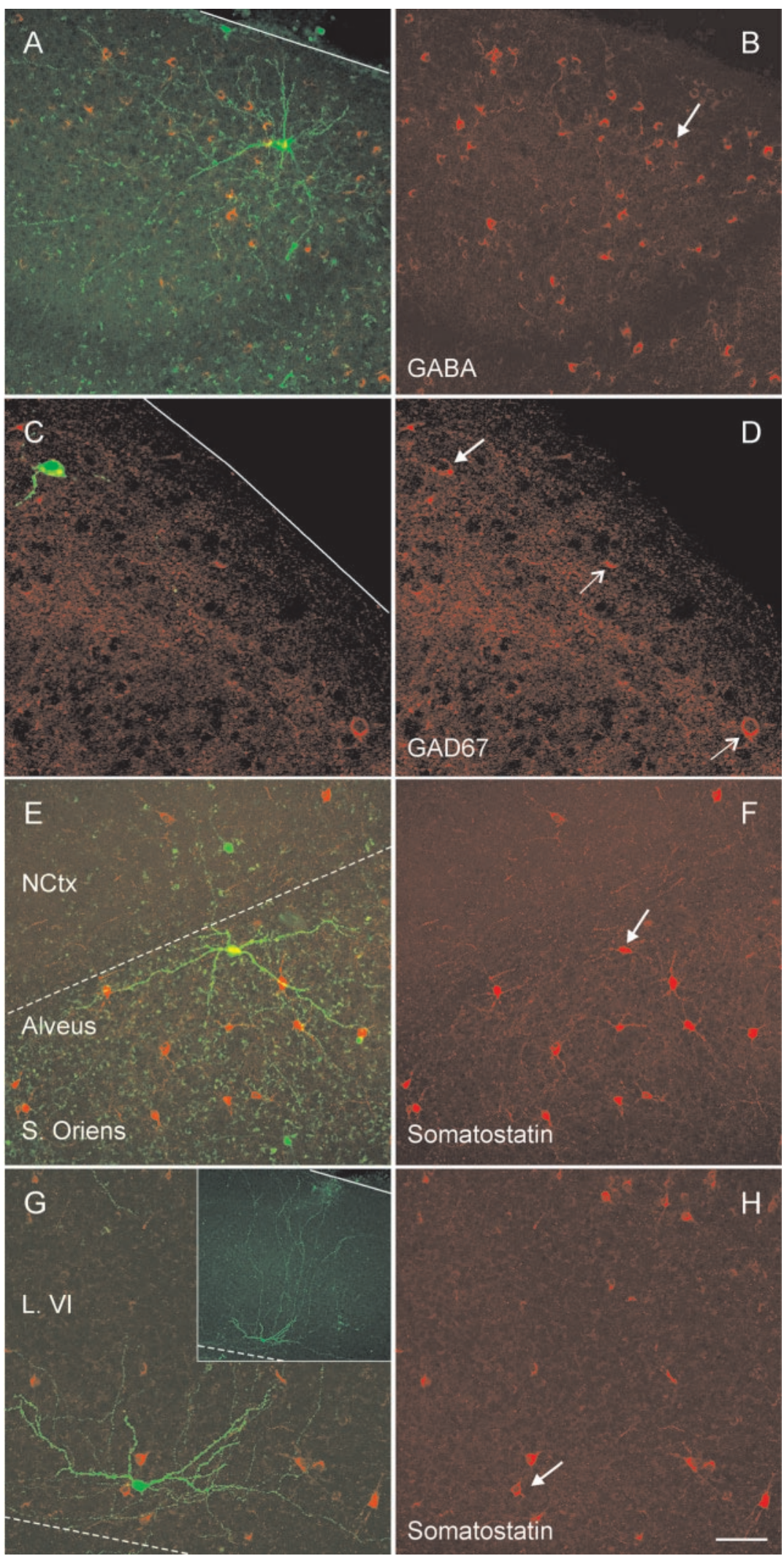

Figure 2. Some, but not all, of the GAD67-GFP-expressing neurons were immunopositive for GABA and GABAergic subtype markers. $A, C, E, G$, Dual-channel confocal images of immunofluorescence (pseudocolored red) and GAD67-GFP fluorescence (pseudocolored green). $B, D, F, H$, The same fields with immunofluorescence only. Immunostaining is for antibodies against $G A B A$ $(A, B), \mathrm{GAD} 67(C, D)$, and somatostatin $(E-H)$. Arrows in $B, D, F$, and $H$ indicate the location of the GFP-expressing neuron shown in $A, C, E$, and $G$, respectively. Note that the GFP-expressing neurons in $A, C$, and $E$ were immunopositive, but the neuron in $G$ was immunonegative. Light arrows in D indicate non-GFP-expressing immunopositive neurons. Scale bar: (in $H$ ) $A, B, E-H, 100 \mu \mathrm{m}$; $C, D, 40 \mu \mathrm{m}$. NCtx, Neocortex; S Oriens, stratum oriens; L. VI, layer 6. Solid and dashed lines are as described in Figure 1. 

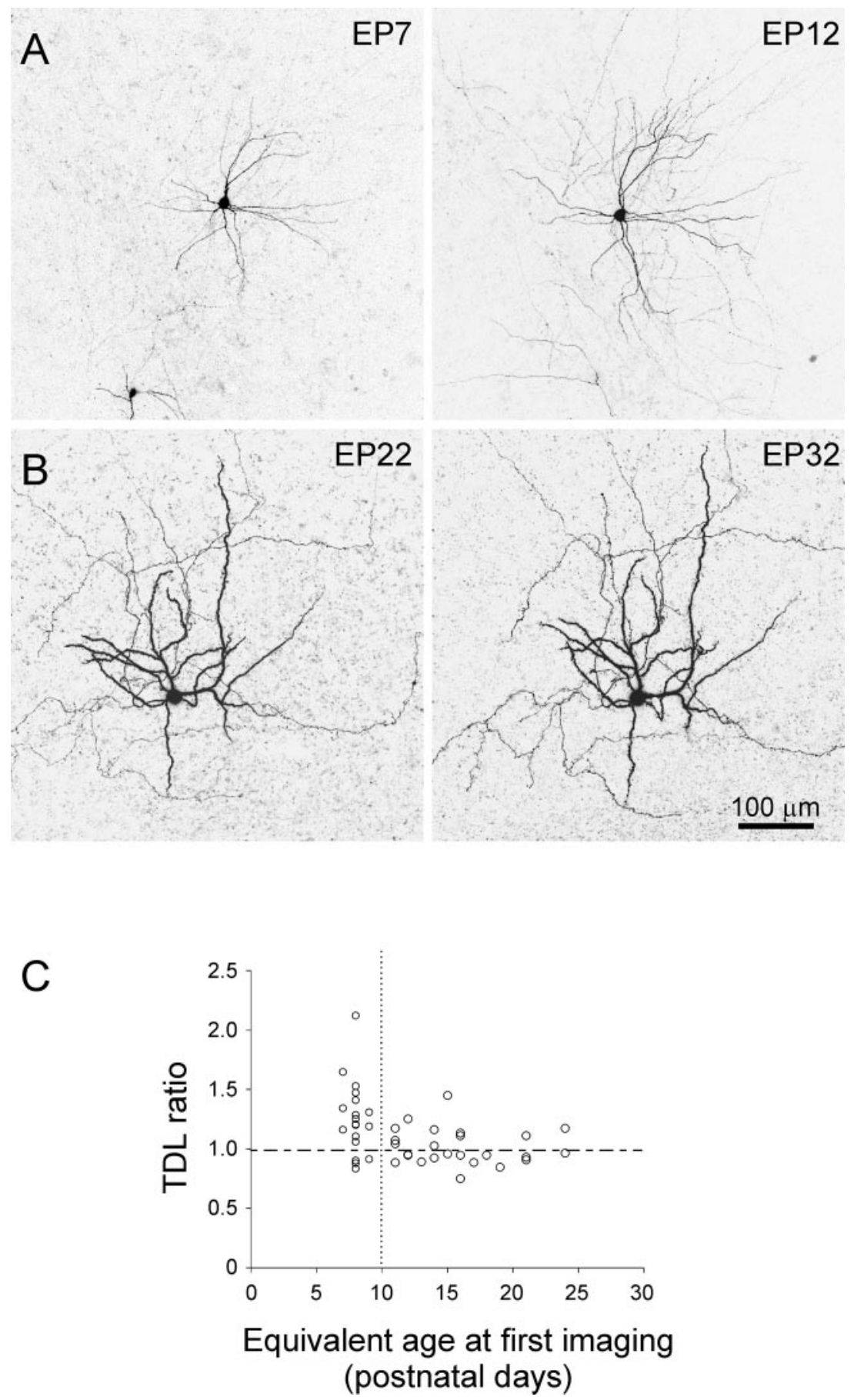

Figure 3. Dendritic growth of GAD67-GFP-expressing neurons in control medium occurred only in younger cultures. $A$, Confocal projection of a representative GAD67-GFP-expressing neuron first imaged at EP7 (left) and again $5 \mathrm{~d}$ later (right). B, Another neuron first imaged at EP22 (left) and again 10 d later (right). Scale bar: (in $B) A, B, 100 \mu \mathrm{m}$. C, TDL growth ratio of all of the neurons in our sample during a $5 \mathrm{~d}$ period in normal medium, plotted against the equivalent age at first imaging. A TDL ratio of 1 (dashed line) indicates no change. Note that dendritic growth was observed in neurons first imaged at EP7-EP9 (left of the vertical dotted line), but there was no change on average in neurons first imaged at later ages (right of the vertical dotted line).

tin, a much smaller molecule commonly used for reconstruction of neuronal morphology after intracellular recordings. Seven GFP-expressing neurons were imaged with a confocal microscope and then injected with biocytin through a patch pipette, processed for the histochemical DAB reaction, and traced with a computerized system. The 3-D dendritic morphology was then compared between reconstructed confocal images and biocytin tracings of the same neuron (Fig. 4). We found that, in older neurons, dendritic reconstructions from GFP and biocytin closely overlapped, after adjusting for shrinkage (Fig. $4 A$ ). In some of the younger neurons, however, GFP tracings from confocal stacks missed a terminal portion of one or two dendritic branches, as evident from comparison with the biocytin image (Fig. $4 B$, top tracing, arrow). This was not caused by insufficient GFP filling, but rather by loss of optical signal from dendritic branches growing deep into the tissue, as illustrated by the $x-z$ projection in Figure $4 B$, bottom tracing, arrow. This situation was easy to identify, because such branches gradually disappeared from view in deeper optical sections; these branches were thereby excluded from analysis in all of the images taken from that particular neuron.

\section{BDNF, but not NT4/5, enhanced dendritic growth in nonpyramidal interneurons}

To investigate the role of BDNF and activity in regulating dendritic growth of nonpyramidal interneurons, we followed the morphological development of individual GAD67-GFP-expressing neurons over a $5 \mathrm{~d}$ period and compared TDL and TDBP ratios between neurons from cultures treated with $200 \mathrm{ng} / \mathrm{ml} \mathrm{BDNF}$, or one of several other treatments, to neurons from untreated control cultures. Representative neurons are illustrated in Figure 5, and a summary plot of all of the experiments is presented in Figure 6. As illustrated in Figure $5 A$, growth in control conditions was modest (note the small rightward shift in the cumulative histogram). As reported above, the mean control TDL ratio (i.e., TDL at day 5 divided by TDL at day 0) was $1.22 \pm 0.07(n=19)$; this change was significantly different from $1(p=0.01)$, but there was no significant change in TDBPs. In contrast, GAD67-GFP-expressing neurons developing in the presence of $\mathrm{BDNF}$ (Fig. 5B) exhibited considerably more growth. The mean TDL ratio in the presence of BDNF was $1.42 \pm 0.05(n=27)$, representing a fractional increase (42\%) nearly double that in control medium (22\%). BDNF also promoted branching of existing dendrites (but not addition of primary dendrites): the mean TDBP ratio in BDNF was $1.25 \pm 0.09$. Both TDL and TDBP ratios in the BDNF group were significantly different from those of the control group ( $p=0.03$ and 0.01 , respectively) (Fig. $6 A, B)$. These results indicate that BDNF promotes dendritic growth and complexity in nonpyramidal neocortical interneurons.

To test whether the growth-promoting effect of BDNF on nonpyramidal, GABAergic interneurons depended on electrical activity, we allowed cultures to develop in the presence of both $200 \mathrm{ng} / \mathrm{ml}$ 


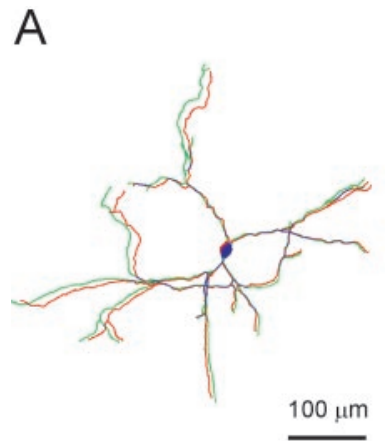

B

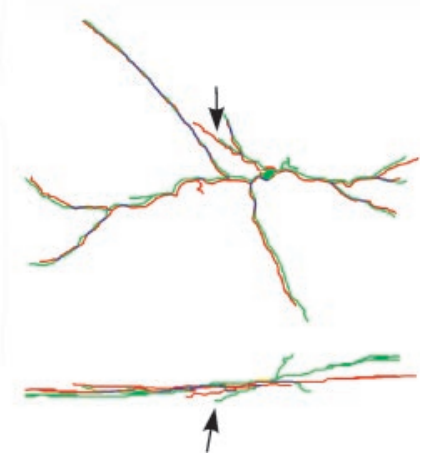

Figure 4. GAD67-GFP fluorescence revealed the full dendritic morphology. GAD67-GFPexpressing neurons were imaged 3 weeks $(A)$ or $3 \mathrm{~d}(B)$ after transfection and then injected with biocytin through a patch pipette. The 3-D reconstructions of the dendritic trees from the confocal images (green) are superimposed on the 3-D reconstructions from the biocytin tracings of the same neurons (red). Note that the GFP fluorescence was at least as extensive as the biocytin labeling, but one secondary dendrite in the younger neuron in $B$ appeared shorter in the confocal image compared with the corresponding biocytin tracing ( $B$, top tracing, arrow). The terminal part of the dendrite was missing from the confocal reconstruction, because it was located deep in the slice, as shown by the $x-z$ image of the same neuron ( $B$, bottom tracing, arrow). Scale bar, $100 \mu \mathrm{m}$ (for both images).

BDNF and $1 \mu \mathrm{M}$ TTX (Fig. 5C). Under these conditions, there was still enhanced dendritic growth in GAD67-GFP-expressing neurons, and the mean TDL ratio was $1.62 \pm 0.11(n=17)$, significantly larger than control ( $p=0.001$ ) (Fig. $6 A$ ) (the increase in TDL ratio compared with BDNF alone was only marginally significant at the $p=0.06$ level). TTX did seem to prevent branching (Fig. $6 B)(p=$ $0.6)$. These data suggest that, unlike the effect of BDNF on pyramidal neurons (McAllister et al., 1996), the enhancing effect of BDNF on dendritic growth of GABAergic interneurons does not require sodium-dependent electrical activity.

To study the role of endogenous BDNF in dendritic development in culture, we compared dendritic growth in control slices with growth in cultures in which endogenous BDNF was neutralized with a high titer $(50 \mu \mathrm{g} / \mathrm{ml})$ of anti-BDNF antibodies, shown to block the effect of endogenous BDNF (Ghosh et al., 1994; Marty, 2000; Seil and Drake-Baumann, 2000), or in which Trk signaling was blocked by $200 \mathrm{~nm}$ Trk signaling inhibitor K252a (Knusel and Hefti, 1992). Under both conditions, dendritic growth was significantly reduced compared with control (Fig. $6 A$ ) (TDL ratios were $1.07 \pm 0.04, n=21, p=0.03$ for anti$\mathrm{BDNF}$; $0.99 \pm 0.05, n=14, p=0.006$ for K252a), indicating that dendritic growth in vitro is regulated by release of endogenous BDNF into the extracellular milieu. Finally, we tested the effect of the neurotrophin NT4/5, which also activates TrkB receptors and is expressed in the cortex during early development (Maisonpierre et al., 1990; Timmusk et al., 1993). NT4/5 (250 ng/ml) had no enhancing effect on dendritic growth or branching of nonpyramidal interneurons; indeed, growth in NT4/5 was significantly reduced (Fig. $6 A)(1.07 \pm 0.05, n=14, p=0.03)$, possibly because NT4/5 competed with endogenous BDNF for TrkB binding sites (Janiga et al., 2000).

\section{Effect of neuronal activity on GABAergic neuron dendritic growth}

Electrical activity can increase several parameters of GABAergic function (Rutherford et al., 1997; Marty et al., 2000), but its effect on dendritic growth of cortical GABAergic neurons was not known. We tested the effect of electrical activity on GAD67-GFP-
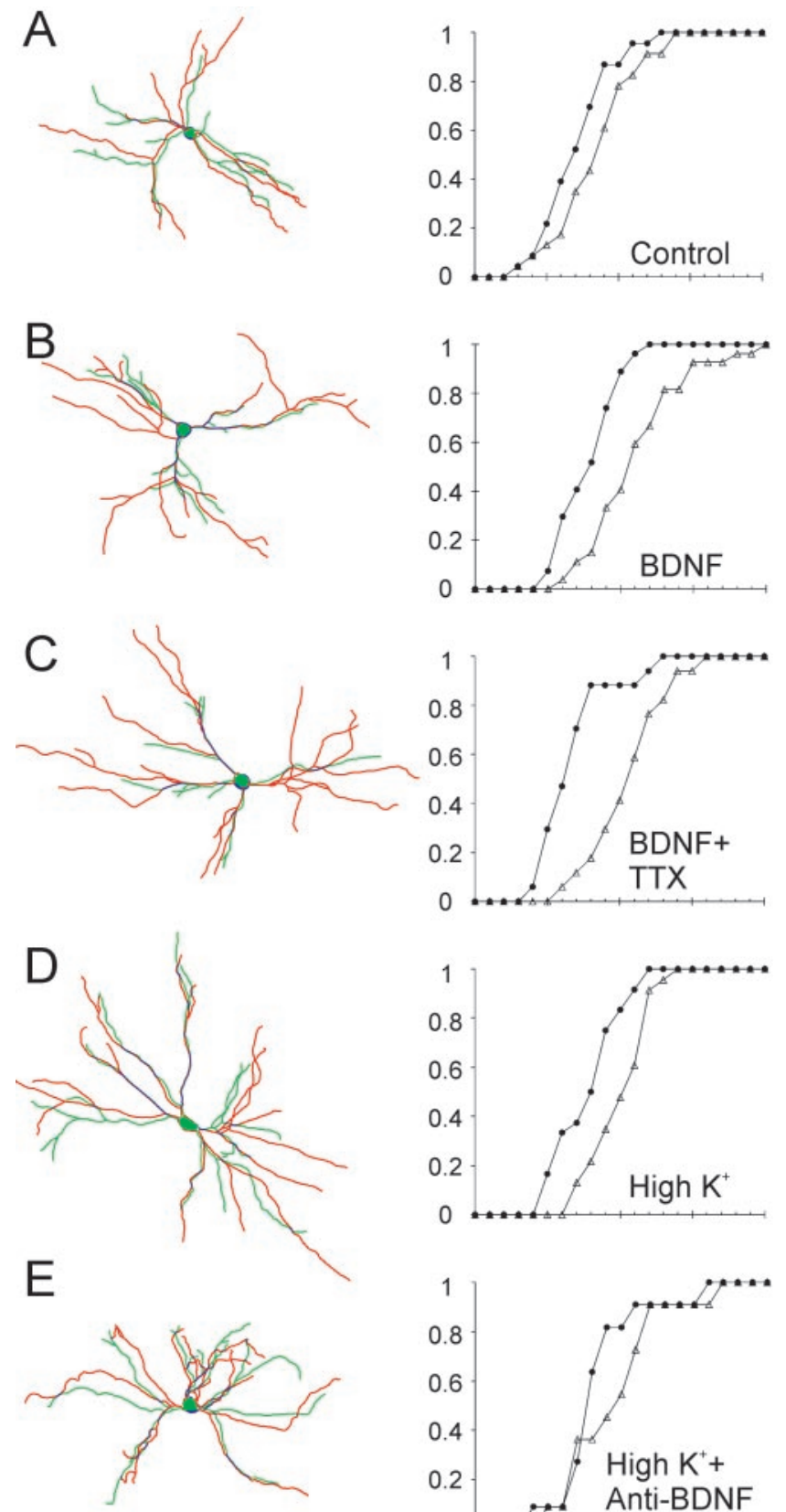

$\overline{100 \mu \mathrm{m}}$

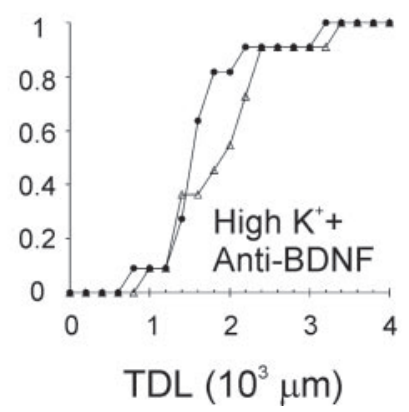

Figure 5. BDNF and KCl enhanced dendritic growth of GAD67-GFP-expressing neurons. Left column, Morphological reconstructions of representative dendritic trees of neurons before (green) and after (red) a $5 \mathrm{~d}$ period in culture medium supplemented by one of the following: no supplement $(A), 200 \mathrm{ng} / \mathrm{ml} \mathrm{BDNF}(B), 200 \mathrm{ng} / \mathrm{ml} \mathrm{BDNF}$ and $1 \mu \mathrm{m} \operatorname{TTX}(C), 10 \mathrm{~mm} \mathrm{KCl}(D)$, or 10 $\mathrm{mm} \mathrm{KCl}$ and $50 \mu \mathrm{g} / \mathrm{ml}$ anti-BDNF $(E)$. Right column, Cumulative histogram of TDL in each treatment group; each data point represents the fraction of neurons in the treatment group with TDL values equal to or smaller than the corresponding $x$ value. $O$, TDL at day 0 (before treatment). $\triangle$, TDL at day 5 (after treatment). Note that the rightward shift of the curves (indicating an overall increase in total dendritic length) was considerably larger in $B, C$, and $D$, compared with control. Scale bar, $100 \mu \mathrm{m}$ (for all images).

expressing neurons by incubating slice cultures in a medium containing $10 \mathrm{~mm} \mathrm{KCl} \mathrm{(Fig.} \mathrm{5D),} \mathrm{which} \mathrm{depolarizes} \mathrm{cortical} \mathrm{neurons}$ and causes action potential firing (Franklin et al., 1995; Tongiorgi et al., 1997; Vaillant et al., 2002). GAD67-GFP neurons cultured in a high $\mathrm{KCl}$ medium exhibited enhanced growth and branching 


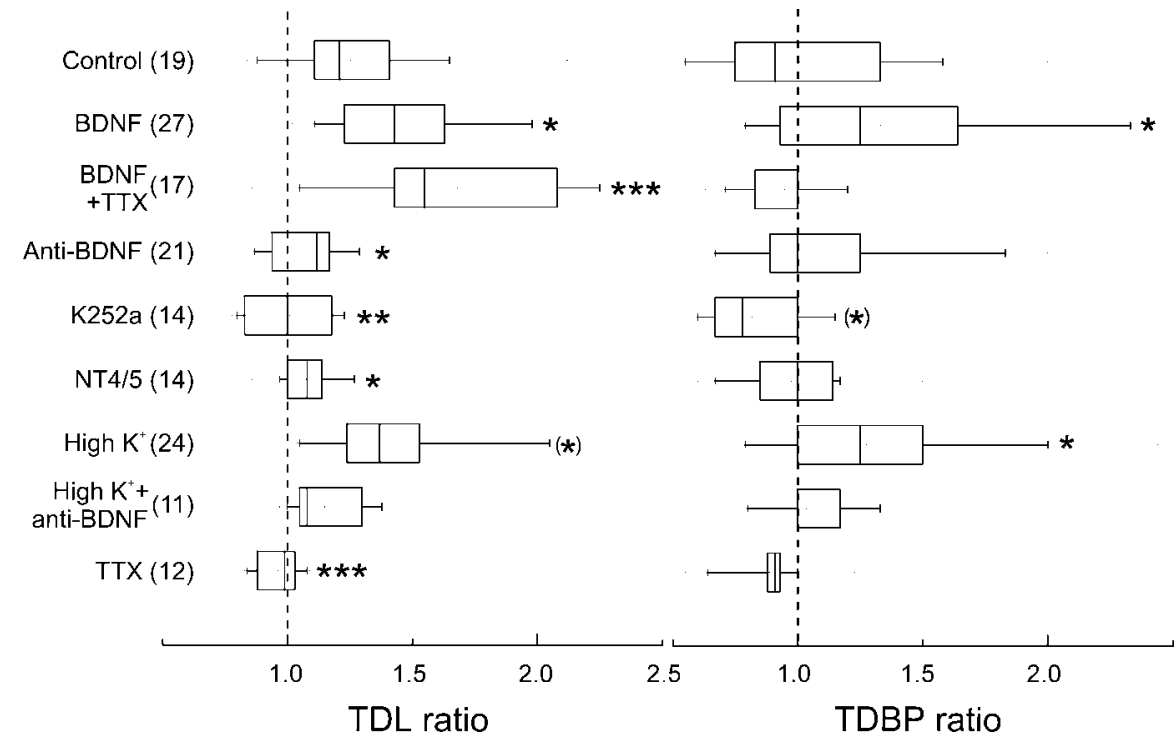

Figure 6. Summary and statistical analysis of the effects of all of the tested treatments on dendritic growth and branching. Ratio change in TDL (left) and TDBPs (right) after $5 \mathrm{~d}$ of the treatment indicated. Each box spans the 25th to 75 th percentile of the data points, with the median represented by a vertical line inside the box; the whiskers span the 5 th to 95 th percentiles. The number of neurons tested is indicated in parentheses after the treatment label. The dashed vertical lines at $x=1$ indicate no change. ${ }^{(*)} p<$ $0.1,{ }^{*} p<0.05,{ }^{* *} p<0.01,{ }^{* * *} p<0.005 ; p$ values are from pairwise comparisons with the control group.

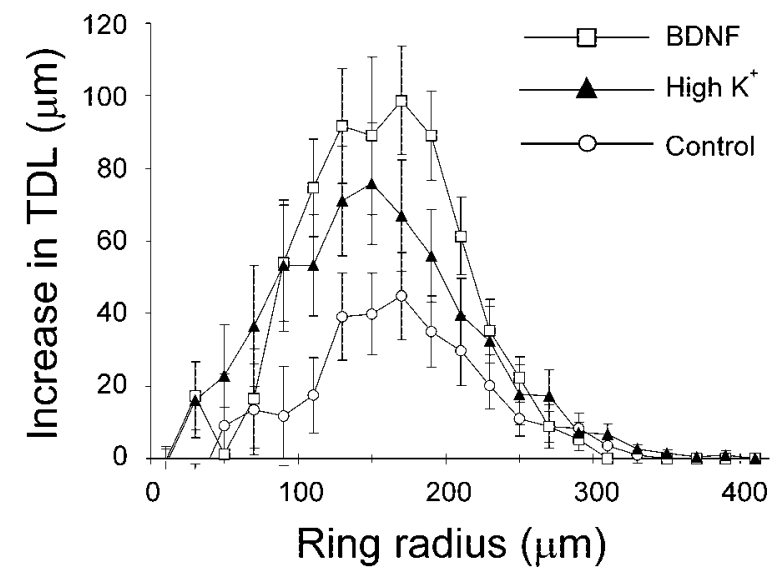

Figure 7. Sholl analysis reveals a similar pattern of dendritic growth in response to BDNF and $\mathrm{KCl}$. Absolute increase in TDL in the control $(\bigcirc)$, high $\mathrm{K}^{+}(\mathbf{\Delta})$, and BDNF $(\square)$ groups was calculated separately for each consecutive, $20 \mu \mathrm{m}$-wide concentric ring, and averaged between neurons. Note that the high $\mathrm{K}^{+}$and BDNF groups exhibited a greater average increase in TDL, compared with control, but the radial extent of the increase was similar among all three groups. Error bars represent SEMs.

compared with control (the mean TDL and TDBP ratios were $1.39 \pm 0.06$ and $1.22 \pm 0.09$, respectively; $n=24)$. The effect on branch points was statistically significant $(p=0.03)$; however, the effect on dendritic length was only marginally so $(p=0.08)$ (Fig. 6), most likely reflecting the modest level of depolarization expected from $10 \mathrm{~mm} \mathrm{KCl}$.

Because electrical activity enhances cortical BDNF levels (Castren et al., 1992; Tongiorgi et al., 1997), the effect of high $\mathrm{K}^{+}$ could have been mediated through activity-dependent release of BDNF. Consistent with this interpretation, the effects of high $\mathrm{KCl}$ on dendritic growth and complexity were not significantly different from the effects of BDNF ( $p=0.76$ and 0.63 , respectively). We tested the dependence of the $\mathrm{KCl}$ effect on released BDNF by neutralizing endogenous extracellular BDNF with anti-BDNF antibodies (Fig. 5E). Dendritic development in a medium sup- plemented with $10 \mathrm{~mm} \mathrm{KCl}$ and $50 \mu \mathrm{g} / \mathrm{ml}$ anti-BDNF was nearly blocked: the TDL ratio was $1.14 \pm 0.05$, and the TDBP ratio was $1.02 \pm 0.06(n=11)$. TDL in high $\mathrm{KCl}$ plus anti-BDNF was very significantly different from growth in $\mathrm{KCl}$ alone $(p=$ 0.002 ), and TDBP was marginally so ( $p=$ 0.07 ), but both were not significantly different from growth and branching in antiBDNF alone $(p=0.3$ and 0.73 , respectively), consistent with the interpretation that the dendritic growth-promoting effect of high $\mathrm{KCl}$ was indeed mediated by BDNF, rather than through an independent, additive pathway (because if the latter was the case, high $\mathrm{KCl}$ should have promoted some growth even in the presence of anti-BDNF).

To test whether growth in normal culture medium is dependent on electrical activity, we cultured slices in the presence of $1 \mu \mathrm{M}$ TTX. Mean TDL ratio in TTX was $0.96 \pm 0.03(n=12)$, very significantly different from control ( $p=0.0002)$ (Fig. $6 A)$, indicating that TTX counteracted the increase in TDL that occurred in normal medium and suggesting that sodium-dependent electrical activity is required for normal dendritic growth in culture.

Finally, to test whether BDNF and depolarization affect dendritic growth in a similar manner, we used Sholl analysis to quantify dendritic growth within consecutive concentric rings around the cell body, and compared Sholl plots showing the increase in dendritic length over a 5 d growth period among control, BDNF, and high $\mathrm{K}^{+}$conditions (Fig. 7). As illustrated, dendritic growth was distributed within a radius of $\sim 300 \mu \mathrm{m}$ around the cell body, with the most growth occurring between 50 and $250 \mu \mathrm{m}$ from the cell body. No major differences were evident between growth patterns in BDNF and high $\mathrm{K}^{+}$, consistent with the conclusion that growth under both conditions was mediated by the same cellular mechanisms.

\section{Discussion}

In this study, we tested whether exogenous BDNF or depolarization by high $\mathrm{KCl}$ enhanced dendritic growth of nonpyramidal cortical interneurons expressing GAD67-GFP in organotypic slice cultures, and whether there was cross-dependency between the effects of BDNF and depolarization. Short-term (minutes to hours) changes in dendritic morphology were tracked previously in living GFP-expressing cortical neurons in vitro (Fischer et al., 1998; Engert and Bonhoeffer, 1999; Horch et al., 1999; MaleticSavatic et al., 1999; Wu et al., 2001) or in vivo (Lendvai et al., 2000), but our study is the first, to our knowledge, to follow the morphological development of the same neocortical neurons over 5 or more days. By imaging the same GFP-expressing neurons at $5 \mathrm{~d}$ intervals, we demonstrated that (1) both BDNF and elevated $\mathrm{K}^{+}$enhanced dendritic growth of nonpyramidal interneurons to the same degree, (2) the effect of BDNF was not dependent on neuronal activity, but (3) the $\mathrm{KCl}$-induced dendritic growth was dependent on release of endogenous BDNF. Together, these observations suggest a role for BDNF in regulating structural and functional maturation of GABAergic interneurons in the developing neocortex and potentially mediating activityinduced dendritic remodeling in the adult. 


\section{BDNF effects in pyramidal and nonpyramidal neocortical interneurons are qualitatively different}

Exogenous or endogenous BDNF, NT3, and NT4/5 have been shown to promote dendritic elongation and branching in neocortical pyramidal cells developing in organotypic cultures (McAllister et al., 1995, 1997; Horch et al., 1999; Niblock et al., 2000; Yacoubian and Lo, 2000), but their effects on nonpyramidal, inhibitory neocortical interneurons were not studied previously. Our results show that activation of TrkB receptors by BDNF, but not by NT4/5, promotes dendritic elongation and branching in developing neocortical nonpyramidal interneurons. Although both BDNF and NT4/5 activate TrkB receptors, the two ligands often affect dendritic growth differentially, even in the same neurons (McAllister et al., 1995; Bosco and Linden, 1999; Steljes et al., 1999).

In our study, a $4 \mathrm{~d}$ treatment with $200 \mathrm{ng} / \mathrm{ml}$ BDNF caused a $40 \%$ increase in TDL of neocortical interneurons. In contrast, a $36 \mathrm{hr}$ treatment with $200 \mathrm{ng} / \mathrm{ml}$ BDNF doubles the TDL and TDBPs of layer 4 pyramidal neurons in the ferret (McAllister et al., 1995). We noted several other differences between the published effects of BDNF on neocortical pyramidal neurons and the effects on nonpyramidal neurons in this study: BDNF did not increase the number of primary dendrites in nonpyramidal neurons, but does so in pyramidal cells (McAllister et al., 1995, 1996; Horch et al., 1999); TTX did not prevent enhancement by BDNF of nonpyramidal cell dendritic growth, but does prevent some of the effects of BDNF on pyramidal cells (McAllister et al., 1996); and BDNF-induced dendritic branches were stable for up to several weeks in nonpyramidal cells, but are highly unstable in pyramidal neurons (Horch et al., 1999). Together, these comparisons suggest that the effects of BDNF on pyramidal and nonpyramidal neurons are qualitatively different, possibly because these two classes of cells express different complements of TrkB receptors or different downstream effectors of TrkB activation.

\section{Depolarization promotes dendritic growth in nonpyramidal neocortical interneurons}

We found that depolarizing neurons by adding $10 \mathrm{~mm} \mathrm{KCl}$ to our organotypic cultures enhanced dendritic growth and branching of nonpyramidal interneurons to a level similar to that induced by $200 \mathrm{ng} / \mathrm{ml}$ BDNF, whereas blocking action potentials with TTX prevented dendritic growth. Therefore, these data suggest that dendritic growth of cortical interneurons in organotypic cultures requires action potentials and can be enhanced by depolarization. KCl-induced enhancement and/or TTX-induced reduction of dendritic length and complexity have been demonstrated previously in some systems (Reitstetter and Yool, 1998; Mertz et al., 2000; Vaillant et al., 2002), but not in others (Riccio and Matthews, 1987; Dalva et al., 1994; Rajan and Cline, 1998). Notably, TTX- or KCl-induced changes in dendritic length were not observed in dissociated cortical neurons in vitro (Kossel et al., 1997; Ramakers et al., 1998). If, as we propose below, the effect of depolarization was mediated by extracellular BDNF, then the failure to observe this effect in dissociated cultures could be attributable to the lower density of neurons in these cultures compared with organotypic slices and to dilution of the released BDNF to ineffective levels.

\section{Depolarization effects are mediated by BDNF released by pyramidal neurons}

Depolarization can promote dendritic growth in a cellautonomous manner, e.g., by activating voltage-gated calcium

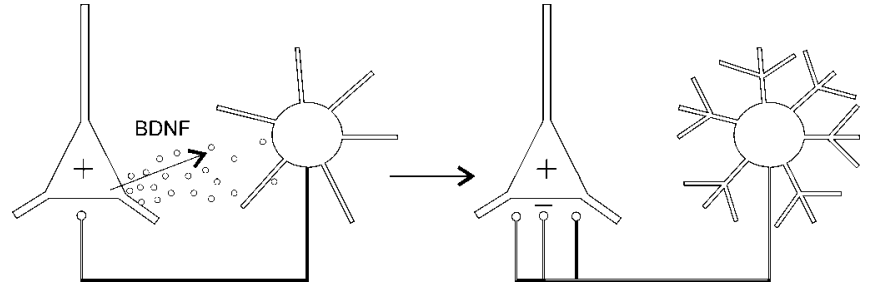

Figure 8. BDNF mediates a feedback loop that maintains a balance between excitation and inhibition. Left, A pyramidal neuron is assumed to be depolarized $(+)$, causing it to increase its firing rate and thereby release more BDNF into the extracellular space, acting on TrkB receptors in a nearby inhibitory neuron. (Although this figure implies that BDNF is released from dendrites, BDNF may also be released from axon terminals.) Right, The released BDNF caused the inhibitory interneuron to ramify its dendritic and axonal arbors and thereby increase its inhibitory effect on the pyramidal cell $(-)$ counteracting the initial depolarization.

channels, followed by calcium-dependent phosphorylation of the dendritic protein MAP2 (microtubule-associated protein 2) (Quinlan and Halpain, 1996; Sanchez et al., 2000; Wu et al., 2001; Vaillant et al., 2002). Alternatively, depolarization can enhance dendritic growth through cell-cell signaling (Matsutani and Yamamoto, 1998; Nedivi et al., 1998). The most parsimonious explanation of our results is that activity-dependent dendritic growth in our experiments was mediated by cell-cell signaling via $\mathrm{BDNF}$, because the $\mathrm{KCl}$-induced growth was prevented by antibodies to BDNF; however, it is difficult to rule out the possibility that $\mathrm{KCl}$ acted via a cell-autonomous pathway, and that BDNF provided only permissive conditions for the activation of this pathway.

In the cerebral cortex, BDNF is expressed only by pyramidal neurons, but TrkB, the preferred receptor for BDNF, is found in both pyramidal cells and GABAergic interneurons (Cellerino and Maffei, 1996; Rocamora et al., 1996; Gorba and Wahle, 1999). BDNF expression and release is induced or enhanced by excitatory synaptic and electrical activity, as has been consistently demonstrated in vitro (Zafra et al., 1990; Ghosh et al., 1994; Wetmore et al., 1994; Goodman et al., 1996; Gorba et al., 1999; Balkowiec and Katz, 2002) and in vivo (Isackson et al., 1991; Castren et al., 1992; Suzuki et al., 1995; Rocamora et al., 1996; Yan et al., 1997; Rossi et al., 1999). We therefore propose that depolarizationinduced synthesis and release of BDNF from pyramidal neurons, either from dendrites (Davies, 1996; Haubensak et al., 1998; Hartmann et al., 2001; Kojima et al., 2001) or from axon terminals (Fawcett et al., 1998; Fawcett et al., 2000; Kohara et al., 2001), activated TrkB receptors on nearby GABAergic neurons and promoted their dendritic growth. In addition, it is possible that depolarization also enhanced TrkB expression on the recipient interneurons themselves (Tongiorgi et al., 1997; Meyer-Franke et al., 1998), causing them to be more responsive to ambient levels of BDNF.

A larger dendritic tree is likely to receive more axodendritic (mostly excitatory) synapses and thus increase electrical activity in the neuron. Moreover, the larger dendritic trees were most likely accompanied by larger (or denser) axonal arbors, as shown previously in dissociated cultures (Vicario-Abejon et al., 1998), although, in our slice cultures, it was difficult to image the axonal arbor in its entirety; a larger axonal arbor is likely to make more inhibitory synapses on postsynaptic targets. Thus, the depolarization-induced increase in network activity would be followed by an increase in synaptic inhibition, bringing activity levels back down. This negative feedback loop is illustrated schematically in Figure 8. 


\section{BDNF and activity-dependent maturation of GABAergic function}

Electrical and synaptic activity in vitro has been shown previously to promote, and blockade of this activity, to reverse, an increase in the level of expression of GABA and GABAergic markers (Marty et al., 1996b; Rutherford et al., 1997) and an increase in the density of GABAergic synapses (Marty et al., 2000; Seil and Drake-Baumann, 2000). Similar to the effects demonstrated here, in the same studies these effects of activity were reproduced by exogenous BDNF and prevented by BDNF blockers, and/or, conversely, the effects of activity blockade were reproduced by BDNF blockers and prevented by exogenous BDNF, indicating that activity exerts its effects via BDNF-TrkB signaling. Various other aspects of GABAergic maturation were shown to be dependent on or enhanced by BDNF in vitro (Seil et al., 1994; Widmer and Hefti, 1994; Murphy et al., 1998; Yamada et al., 2002) and in vivo (Nawa et al., 1994; Huang et al., 1999; Aguado et al., 2003). Thus, BDNF may play a major role in regulating the maturation of the GABAergic inhibitory system and may act during development to keep GABAergic inhibition in step with the level of excitatory activity, thereby maintaining activity homeostasis in the network (Marty et al., 1997; Bolton et al., 2000; Turrigiano and Nelson, 2000). In the adult, BDNF may also mediate the effects of other physiological parameters, such as hormonal state, on dendritic morphology (Barbany and Persson, 1992; Toran-Allerand, 1996; Murphy et al., 1998). Finally, in addition to its global effect on the balance between excitation and inhibition, BDNF could also act very locally, by virtue of its anterograde or retrograde release and uptake in synapses (Altar et al., 1997; Fawcett et al., 1998; Hartmann et al., 2001; Kohara et al., 2001) and the dendritic targeting of its receptor protein and mRNA (Fryer et al., 1996; Tongiorgi et al., 1997), to promote growth of dendritic branches receiving active synapses at the expense of branches opposite less active synapses. Thus, BDNF could mediate dynamic sculpting of dendritic fields, providing a morphological substrate for activitydependent plasticity of neuronal circuits, both during development and in adult learning.

\section{References}

Agmon A, O'Dowd DK (1992) NMDA receptor-mediated currents are prominent in the thalamocortical synaptic response before maturation of inhibition. J Neurophysiol 68:345-349.

Agmon A, Hollrigel G, O’Dowd DK (1996) Functional GABAergic synaptic connection in neonatal mouse barrel cortex. J Neurosci 16:4684-4695.

Aguado F, Carmona MA, Pozas E, Aguilo A, Martinez-Guijarro FJ, Alcantara S, Borrell V, Yuste R, Ibanez CF, Soriano E (2003) BDNF regulates spontaneous correlated activity at early developmental stages by increasing synaptogenesis and expression of the $\mathrm{K}^{+} / \mathrm{Cl}^{-}$co-transporter KCC2. Development 130:1267-1280.

Alcantara S, Ferrer I, Soriano E (1993) Postnatal development of parvalbumin and calbindin D28K immunoreactivities in the cerebral cortex of the rat. Anat Embryol (Berl) 188:63-73.

Altar CA, Cai N, Bliven T, Juhasz M, Conner JM, Acheson AL, Lindsay RM, Wiegand SJ (1997) Anterograde transport of brain-derived neurotrophic factor and its role in the brain. Nature 389:856-860.

Balcar VJ, Zetzsche T, Wolff JR (1992) Glutamate decarboxylase in developing rat neocortex: does it correlate with the differentiation of GABAergic neurons and synapses? Neurochem Res 17:253-260.

Balkowiec A, Katz DM (2002) Cellular mechanisms regulating activitydependent release of native brain-derived neurotrophic factor from hippocampal neurons. J Neurosci 22:10399-10407.

Bao S, Chen L, Qiao X, Thompson RF (1999) Transgenic brain-derived neurotrophic factor modulates a developing cerebellar inhibitory synapse. Learn Mem 6:276-283.

Barbany G, Persson H (1992) Regulation of neurotrophin mRNA expression in the rat brain by glucocorticoids. Eur J Neurosci 4:396-403.

Bolton MM, Lo DC, Sherwood NT (2000) Long-term regulation of excita- tory and inhibitory synaptic transmission in hippocampal cultures by brain-derived neurotrophic factor. Prog Brain Res 128:203-218.

Bonhoeffer T (1996) Neurotrophins and activity-dependent development of the neocortex. Curr Opin Neurobiol 6:119-126.

Bosco A, Linden R (1999) BDNF and NT-4 differentially modulate neurite outgrowth in developing retinal ganglion cells. J Neurosci Res 57:759-769.

Carmona MA, Martinez A, Soler A, Blasi J, Soriano E, Aguado F (2003) $\mathrm{Ca}^{2+}$-evoked synaptic transmission and neurotransmitter receptor levels are impaired in the forebrain of $t r k b(-/-)$ mice. Mol Cell Neurosci 22:210-226.

Castren E, Zafra F, Thoenen H, Lindholm D (1992) Light regulates expression of brain-derived neurotrophic factor mRNA in rat visual cortex. Proc Natl Acad Sci USA 89:9444-9448.

Cellerino A, Maffei L (1996) The action of neurotrophins in the development and plasticity of the visual cortex. Prog Neurobiol [Erratum (1996) 50:333] 49:53-71.

Cohen AS, Lin DD, Coulter DA (2000) Protracted postnatal development of inhibitory synaptic transmission in rat hippocampal area CA1 neurons. J Neurophysiol 84:2465-2476.

Dalva MB, Ghosh A, Shatz CJ (1994) Independent control of dendritic and axonal form in the developing lateral geniculate nucleus. J Neurosci 14:3588-3602.

Davies AM (1996) Paracrine and autocrine actions of neurotrophic factors. Neurochem Res 21:749-753.

De Felipe J, Marco P, Fairen A, Jones EG (1997) Inhibitory synaptogenesis in mouse somatosensory cortex. Cereb Cortex 7:619-634.

de Lecea L, del Rio JA, Soriano E (1995) Developmental expression of parvalbumin mRNA in the cerebral cortex and hippocampus of the rat. Brain Res Mol Brain Res 32:1-13.

del Rio JA, de Lecea L, Ferrer I, Soriano E (1994) The development of parvalbumin-immunoreactivity in the neocortex of the mouse. Brain Res Dev Brain Res 81:247-259.

Engert F, Bonhoeffer T (1999) Dendritic spine changes associated with hippocampal long-term synaptic plasticity. Nature 399:66-70.

Fawcett JP, Bamji SX, Causing CG, Aloyz R, Ase AR, Reader TA, McLean JH, Miller FD (1998) Functional evidence that BDNF is an anterograde neuronal trophic factor in the CNS. J Neurosci 18:2808-2821.

Fawcett JP, Alonso-Vanegas MA, Morris SJ, Miller FD, Sadikot AF, Murphy RA (2000) Evidence that brain-derived neurotrophic factor from presynaptic nerve terminals regulates the phenotype of calbindin-containing neurons in the lateral septum. J Neurosci 20:274-282.

Fischer M, Kaech S, Knutti D, Matus A (1998) Rapid actin-based plasticity in dendritic spines. Neuron 20:847-854.

Franklin JL, Sanz-Rodriguez C, Juhasz A, Deckwerth TL, Johnson Jr EM (1995) Chronic depolarization prevents programmed death of sympathetic neurons in vitro but does not support growth: requirement for $\mathrm{Ca}^{2+}$ influx but not Trk activation. J Neurosci 15:643-664.

Freund TF, Buzsaki G (1996) Interneurons of the hippocampus. Hippocampus 6:347-470.

Fryer RH, Kaplan DR, Feinstein SC, Radeke MJ, Grayson DR, Kromer LF (1996) Developmental and mature expression of full-length and truncated TrkB receptors in the rat forebrain. J Comp Neurol 374:21-40.

Ghosh A, Carnahan J, Greenberg ME (1994) Requirement for BDNF in activity-dependent survival of cortical neurons. Science 263:1618-1623.

Good PI (1999) Resampling methods. Boston: Birkhauser.

Goodman LJ, Valverde J, Lim F, Geschwind MD, Federoff HJ, Geller AI, Hefti F (1996) Regulated release and polarized localization of brain-derived neurotrophic factor in hippocampal neurons. Mol Cell Neurosci 7:222-238.

Gorba T, Wahle P (1999) Expression of TrkB and TrkC but not BDNF mRNA in neurochemically identified interneurons in rat visual cortex in vivo and in organotypic cultures. Eur J Neurosci 11:1179-1190.

Gorba T, Klostermann O, Wahle P (1999) Development of neuronal activity and activity-dependent expression of brain-derived neurotrophic factor mRNA in organotypic cultures of rat visual cortex. Cereb Cortex 9:864-877.

Guo Y, Kaplan IV, Cooper NG, Mower GD (1997) Expression of two forms of glutamic acid decarboxylase (GAD67 and GAD65) during postnatal development of the cat visual cortex. Brain Res Dev Brain Res 103:127-141.

Hartmann M, Heumann R, Lessmann V (2001) Synaptic secretion of BDNF 
after high-frequency stimulation of glutamatergic synapses. EMBO J 20:5887-5897.

Haubensak W, Narz F, Heumann R, Lessmann V (1998) BDNF-GFP containing secretory granules are localized in the vicinity of synaptic junctions of cultured cortical neurons. J Cell Sci 111:1483-1493.

Henneberger C, Juttner R, Rothe T, Grantyn R (2002) Postsynaptic action of BDNF on GABAergic synaptic transmission in the superficial layers of the mouse superior colliculus. J Neurophysiol 88:595-603.

Horch HW, Kruttgen A, Portbury SD, Katz LC (1999) Destabilization of cortical dendrites and spines by BDNF. Neuron 23:353-364.

Houser CR, Hendry SH, Jones EG, Vaughn JE (1983) Morphological diversity of immunocytochemically identified GABA neurons in the monkey sensory-motor cortex. J Neurocytol 12:617-638.

Huang ZJ, Kirkwood A, Pizzorusso T, Porciatti V, Morales B, Bear MF, Maffei L, Tonegawa S (1999) BDNF regulates the maturation of inhibition and the critical period of plasticity in mouse visual cortex. Cell 98:739-755.

Isackson PJ, Huntsman MM, Murray KD, Gall CM (1991) BDNF mRNA expression is increased in adult rat forebrain after limbic seizures: temporal patterns of induction distinct from NGF. Neuron 6:937-948.

Janiga TA, Rind HB, von Bartheld CS (2000) Differential effects of the trophic factors BDNF, NT-4, GDNF, and IGF-I on the isthmo-optic nucleus in chick embryos. J Neurobiol 43:289-303.

Jin X, Mathers PH, Szabo G, Katarova Z, Agmon A (2001) Vertical bias in dendritic trees of non-pyramidal neocortical neurons expressing GAD67GFP in vitro. Cereb Cortex 11:666-678.

Katarova Z, Mugnaini E, Sekerkova G, Mann JR, Aszodi A, Bosze Z, Greenspan R, Szabo G (1998) Regulation of cell-type specific expression of lac $Z$ by the $5^{\prime}$-flanking region of mouse GAD67 gene in the central nervous system of transgenic mice. Eur J Neurosci 10:989-999.

Kawaguchi Y, Kubota Y (1996) Physiological and morphological identification of somatostatin- or vasoactive intestinal polypeptide-containing cells among GABAergic cell subtypes in rat frontal cortex. J Neurosci 16:2701-2715.

Kawaguchi Y, Kubota Y (1997) GABAergic cell subtypes and their synaptic connections in rat frontal cortex. Cereb Cortex 7:476-486.

Kirkwood A, Bear MF (1994) Hebbian synapses in visual cortex. J Neurosci 14:1634-1645.

Knusel B, Hefti F (1992) K-252 compounds: modulators of neurotrophin signal transduction. J Neurochem 59:1987-1996.

Kohara K, Kitamura A, Morishima M, Tsumoto T (2001) Activitydependent transfer of brain-derived neurotrophic factor to postsynaptic neurons. Science 291:2419-2423.

Kojima M, Takei N, Numakawa T, Ishikawa Y, Suzuki S, Matsumoto T, Katoh-Semba R, Nawa H, Hatanaka H (2001) Biological characterization and optical imaging of brain-derived neurotrophic factor-green fluorescent protein suggest an activity-dependent local release of brainderived neurotrophic factor in neurites of cultured hippocampal neurons. J Neurosci Res 64:1-10.

Komatsu Y, Iwakiri M (1991) Postnatal development of neuronal connections in cat visual cortex studied by intracellular recording in slice preparation. Brain Res 540:14-24.

Kossel AH, Williams CV, Schweizer M, Kater SB (1997) Afferent innervation influences the development of dendritic branches and spines via both activity-dependent and non-activity-dependent mechanisms. J Neurosci 17:6314-6324.

Kubota Y, Hattori R, Yui Y (1994) Three distinct subpopulations of GABAergic neurons in rat frontal agranular cortex. Brain Res 649:159-173.

Lendvai B, Stern EA, Chen B, Svoboda K (2000) Experience-dependent plasticity of dendritic spines in the developing rat barrel cortex in vivo. Nature 404:876-881.

Lu B (2003) BDNF and activity-dependent synaptic modulation. Learn Mem 10:86-98.

Maisonpierre PC, Belluscio L, Friedman B, Alderson RF, Wiegand SJ, Furth ME, Lindsay RM, Yancopoulos GD (1990) NT-3, BDNF, and NGF in the developing rat nervous system: parallel as well as reciprocal patterns of expression. Neuron 5:501-509.

Maletic-Savatic M, Malinow R, Svoboda K (1999) Rapid dendritic morphogenesis in CAl hippocampal dendrites induced by synaptic activity. Science 283:1923-1927.

Manly BFJ (1997) Randomization, bootstrap and Monte Carlo methods in biology, Ed 2. Boca Raton, FL: Chapman \& Hall/CRC.
Marty S (2000) Differences in the regulation of neuropeptide Y, somatostatin and parvalbumin levels in hippocampal interneurons by neuronal activity and BDNF. Prog Brain Res 128:193-202.

Marty S, Carroll P, Cellerino A, Castren E, Staiger V, Thoenen H, Lindholm D (1996a) Brain-derived neurotrophic factor promotes the differentiation of various hippocampal nonpyramidal neurons, including Cajal-Retzius cells, in organotypic slice cultures. J Neurosci 16:675-687.

Marty S, Berninger B, Carroll P, Thoenen H (1996b) GABAergic stimulation regulates the phenotype of hippocampal interneurons through the regulation of brain-derived neurotrophic factor. Neuron 16:565-570.

Marty S, Berzaghi Mda P, Berninger B (1997) Neurotrophins and activitydependent plasticity of cortical interneurons. Trends Neurosci 20:198-202.

Marty S, Wehrle R, Sotelo C (2000) Neuronal activity and brain-derived neurotrophic factor regulate the density of inhibitory synapses in organotypic slice cultures of postnatal hippocampus. J Neurosci 20:8087-8095.

Marty S, Wehrle R, Alvarez-Leefmans FJ, Gasnier B, Sotelo C (2002) Postnatal maturation of $\mathrm{Na}^{+}, \mathrm{K}^{+}, 2 \mathrm{Cl}^{-}$cotransporter expression and inhibitory synaptogenesis in the rat hippocampus: an immunocytochemical analysis. Eur J Neurosci 15:233-245.

Massengill JL, Smith MA, Son DI, O’Dowd DK (1997) Differential expression of K4-AP currents and Kv3.1 potassium channel transcripts in cortical neurons that develop distinct firing phenotypes. J Neurosci 17:3136-3147.

Matsutani S, Yamamoto N (1998) GABAergic neuron-to-astrocyte signaling regulates dendritic branching in coculture. J Neurobiol 37:251-264.

McAllister AK, Lo DC, Katz LC (1995) Neurotrophins regulate dendritic growth in developing visual cortex. Neuron 15:791-803.

McAllister AK, Katz LC, Lo DC (1996) Neurotrophin regulation of cortical dendritic growth requires activity. Neuron 17:1057-1064.

McAllister AK, Katz LC, Lo DC (1997) Opposing roles for endogenous BDNF and NT-3 in regulating cortical dendritic growth. Neuron 18:767-778.

McAllister AK, Katz LC, Lo DC (1999) Neurotrophins and synaptic plasticity. Annu Rev Neurosci 22:295-318.

Meinecke DL, Peters A (1987) GABA immunoreactive neurons in rat visual cortex. J Comp Neurol 261:388-404.

Mertz K, Koscheck T, Schilling K (2000) Brain-derived neurotrophic factor modulates dendritic morphology of cerebellar basket and stellate cells: an in vitro study. Neuroscience 97:303-310.

Meyer-Franke A, Wilkinson GA, Kruttgen A, Hu M, Munro E, Hanson Jr MG, Reichardt LF, Barres BA (1998) Depolarization and cAMP elevation rapidly recruit TrkB to the plasma membrane of CNS neurons. Neuron 21:681-693.

Micheva KD, Beaulieu C (1995) Postnatal development of GABA neurons in the rat somatosensory barrel cortex: a quantitative study. Eur J Neurosci 7:419-430.

Micheva KD, Beaulieu C (1996) Quantitative aspects of synaptogenesis in the rat barrel field cortex with special reference to GABA circuitry. J Comp Neurol 373:340-354.

Miller MW (1986) Maturation of rat visual cortex. III. Postnatal morphogenesis and synaptogenesis of local circuit neurons. Brain Res 390:271-285.

Mugnaini E, Oertel WH (1985) An atlas of the distribution of GABAergic neurons and terminals in the rat CNS as revealed by GAD immunohistochemistry. In: Handbook of chemical neuroanatomy, Vol 4 (Bjorklund A, Hokfelt T, eds), pp. 436-608. Amsterdam: Elsevier.

Murphy DD, Cole NB, Segal M (1998) Brain-derived neurotrophic factor mediates estradiol-induced dendritic spine formation in hippocampal neurons. Proc Natl Acad Sci USA 95:11412-11417.

Nawa H, Pelleymounter MA, Carnahan J (1994) Intraventricular administration of BDNF increases neuropeptide expression in newborn rat brain. J Neurosci 14:3751-3765.

Nedivi E, Wu GY, Cline HT (1998) Promotion of dendritic growth by CPG15, an activity-induced signaling molecule. Science 281:1863-1866.

Niblock MM, Brunso-Bechtold JK, Riddle DR (2000) Insulin-like growth factor I stimulates dendritic growth in primary somatosensory cortex. J Neurosci 20:4165-4176.

Olofsdotter K, Lindvall O, Asztely F (2000) Increased synaptic inhibition in dentate gyrus of mice with reduced levels of endogenous brain-derived neurotrophic factor. Neuroscience 101:531-539.

Pow DV, Wright LL, Vaney DI (1995) The immunocytochemical detection 
of amino-acid neurotransmitters in paraformaldehyde-fixed tissues. J Neurosci Methods 56:115-123.

Prieto JJ, Peterson BA, Winer JA (1994) Morphology and spatial distribution of GABAergic neurons in cat primary auditory cortex (AI). J Comp Neurol 344:349-382.

Quinlan EM, Halpain S (1996) Emergence of activity-dependent, bidirectional control of microtubule-associated protein MAP2 phosphorylation during postnatal development. J Neurosci 16:7627-7637.

Rajan I, Cline HT (1998) Glutamate receptor activity is required for normal development of tectal cell dendrites in vivo. J Neurosci 18:7836-7846.

Ramakers GJ, Winter J, Hoogland TM, Lequin MB, van Hulten P, van Pelt J, Pool CW (1998) Depolarization stimulates lamellipodia formation and axonal but not dendritic branching in cultured rat cerebral cortex neurons. Brain Res Dev Brain Res 108:205-216.

Ramoa AS, McCormick DA (1994) Enhanced activation of NMDA receptor responses at the immature retinogeniculate synapse. J Neurosci 14:2098-2105.

Reitstetter R, Yool AJ (1998) Morphological consequences of altered calcium-dependent transmembrane signaling on the development of cultured cerebellar Purkinje neurons. Brain Res Dev Brain Res 107:165-167.

Ren JQ, Aika Y, Heizmann CW, Kosaka T (1992) Quantitative analysis of neurons and glial cells in the rat somatosensory cortex, with special reference to GABAergic neurons and parvalbumin-containing neurons. Exp Brain Res 92:1-14.

Riccio RV, Matthews MA (1987) Effects of intraocular tetrodotoxin on the postnatal development of the dorsal lateral geniculate nucleus of the rat: a Golgi analysis. J Neurosci Res 17:440 - 451.

Richardson CA, Leitch B (2002) Cerebellar Golgi, Purkinje, and basket cells have reduced $\gamma$-aminobutyric acid immunoreactivity in stargazer mutant mice. J Comp Neurol 453:85-99.

Rico B, Xu B, Reichardt LF (2002) TrkB receptor signaling is required for establishment of GABAergic synapses in the cerebellum. Nat Neurosci 5:225-233.

Rocamora N, Welker E, Pascual M, Soriano E (1996) Upregulation of BDNF mRNA expression in the barrel cortex of adult mice after sensory stimulation. J Neurosci 16:4411-4419.

Rossi FM, Bozzi Y, Pizzorusso T, Maffei L (1999) Monocular deprivation decreases brain-derived neurotrophic factor immunoreactivity in the rat visual cortex. Neuroscience 90:363-368.

Rothman KJ (1990) No adjustments are needed for multiple comparisons. Epidemiology 1:43-46.

Rozas C, Frank H, Heynen AJ, Morales B, Bear MF, Kirkwood A (2001) Developmental inhibitory gate controls the relay of activity to the superficial layers of the visual cortex. J Neurosci 21:6791-6801.

Rutherford LC, DeWan A, Lauer HM, Turrigiano GG (1997) Brain-derived neurotrophic factor mediates the activity-dependent regulation of inhibition in neocortical cultures. J Neurosci 17:4527-4535.

Sanchez C, Diaz-Nido J, Avila J (2000) Phosphorylation of microtubuleassociated protein 2 (MAP2) and its relevance for the regulation of the neuronal cytoskeleton function. Prog Neurobiol 61:133-168.

Savitz DA, Olshan AF (1998) Describing data requires no adjustment for multiple comparisons: a reply from Savitz and Olshan. Am J Epidemiol 147:813-814; discussion 815.

Seil FJ, Drake-Baumann R (2000) TrkB receptor ligands promote activitydependent inhibitory synaptogenesis. J Neurosci 20:5367-5373.

Seil FJ, Drake-Baumann R, Leiman AL, Herndon RM, Tiekotter KL (1994) Morphological correlates of altered neuronal activity in organotypic cerebellar cultures chronically exposed to anti-GABA agents. Brain Res Dev Brain Res 77:123-132.

Spirou GA, Berrebi AS (1997) Glycine immunoreactivity in the lateral nucleus of the trapezoid body of the cat. J Comp Neurol 383:473-488.

Steljes TP, Kinoshita Y, Wheeler EF, Oppenheim RW, von Bartheld CS (1999) Neurotrophic factor regulation of developing avian oculomotor neurons: differential effects of BDNF and GDNF. J Neurobiol 41:295-315.
Stoppini L, Buchs PA, Muller D (1991) A simple method for organotypic cultures of nervous tissue. J Neurosci Methods 37:173-182.

Sutor B, Luhmann HJ (1995) Development of excitatory and inhibitory postsynaptic potentials in the rat neocortex. Perspect Dev Neurobiol 2:409-419.

Suzuki F, Junier MP, Guilhem D, Sorensen JC, Onteniente B (1995) Morphogenetic effect of kainate on adult hippocampal neurons associated with a prolonged expression of brain-derived neurotrophic factor. Neuroscience 64:665-674.

Szabo G, Katarova Z, Kortvely E, Greenspan RJ, Urban Z (1996) Structure and the promoter region of the mouse gene encoding the $67-\mathrm{kD}$ form of glutamic acid decarboxylase. DNA Cell Biol 15:1081-1091.

Thoenen H (1995) Neurotrophins and neuronal plasticity. Science 270:593-598.

Timmusk T, Belluardo N, Metsis M, Persson H (1993) Widespread and developmentally regulated expression of neurotrophin-4 mRNA in rat brain and peripheral tissues. Eur J Neurosci 5:605-613.

Tongiorgi E, Righi M, Cattaneo A (1997) Activity-dependent dendritic targeting of BDNF and TrkB mRNAs in hippocampal neurons. J Neurosci 17:9492-9505.

Toran-Allerand CD (1996) Mechanisms of estrogen action during neural development: mediation by interactions with the neurotrophins and their receptors? J Steroid Biochem Mol Biol 56:169-178.

Turrigiano GG, Nelson SB (2000) Hebb and homeostasis in neuronal plasticity. Curr Opin Neurobiol 10:358-364.

Vaillant AR, Zanassi P, Walsh GS, Aumont A, Alonso A, Miller FD (2002) Signaling mechanisms underlying reversible, activity-dependent dendrite formation. Neuron 34:985-998.

Ventimiglia R, Mather PE, Jones BE, Lindsay RM (1995) The neurotrophins BDNF, NT-3 and NT-4/5 promote survival and morphological and biochemical differentiation of striatal neurons in vitro. Eur J Neurosci 7:213-222.

Vicario-Abejon C, Collin C, McKay RD, Segal M (1998) Neurotrophins induce formation of functional excitatory and inhibitory synapses between cultured hippocampal neurons. J Neurosci 18:7256-7271.

Vogt Weisenhorn DM, Celio MR, Rickmann M (1998) The onset of parvalbumin-expression in interneurons of the rat parietal cortex depends upon extrinsic factor(s). Eur J Neurosci 10:1027-1036.

Wahle P (1993) Differential regulation of substance P and somatostatin in Martinotti cells of the developing cat visual cortex. J Comp Neurol 329:519-538.

Wells JE, Porter JT, Agmon A (2000) GABAergic inhibition suppresses paroxysmal network activity in the neonatal rodent hippocampus and neocortex. J Neurosci 20:8822-8830.

Wetmore C, Olson L, Bean AJ (1994) Regulation of brain-derived neurotrophic factor (BDNF) expression and release from hippocampal neurons is mediated by non-NMDA type glutamate receptors. J Neurosci 14:1688-1700.

Widmer HR, Hefti F (1994) Stimulation of GABAergic neuron differentiation by NT- $4 / 5$ in cultures of rat cerebral cortex. Brain Res Dev Brain Res $80: 279-284$.

Wu GY, Deisseroth K, Tsien RW (2001) Spaced stimuli stabilize MAPK pathway activation and its effects on dendritic morphology. Nat Neurosci 4:151-158.

Yacoubian TA, Lo DC (2000) Truncated and full-length TrkB receptors regulate distinct modes of dendritic growth. Nat Neurosci 3:342-349.

Yamada MK, Nakanishi K, Ohba S, Nakamura T, Ikegaya Y, Nishiyama N, Matsuki N (2002) Brain-derived neurotrophic factor promotes the maturation of GABAergic mechanisms in cultured hippocampal neurons. J Neurosci 22:7580-7585.

Yan Q, Rosenfeld RD, Matheson CR, Hawkins N, Lopez OT, Bennett L, Welcher AA (1997) Expression of brain-derived neurotrophic factor protein in the adult rat central nervous system. Neuroscience 78:431-448.

Zafra F, Hengerer B, Leibrock J, Thoenen H, Lindholm D (1990) Activity dependent regulation of BDNF and NGF mRNAs in the rat hippocampus is mediated by non-NMDA glutamate receptors. EMBO J 9:3545-3550. 